\title{
0-Hecke algebra action on the Stanley-Reisner ring of the Boolean algebra
}

\author{
Jia Huang $\left.\right|^{*}$ \\ School of Mathematics, University of Minnesota, Minneapolis, MN 55455, USA
}

\begin{abstract}
We define an action of the 0-Hecke algebra of type A on the Stanley-Reisner ring of the Boolean algebra. By studying this action we obtain a family of multivariate noncommutative symmetric functions, which specialize to the noncommutative Hall-Littlewood symmetric functions and their $(q, t)$-analogues introduced by Bergeron and Zabrocki. We also obtain multivariate quasisymmetric function identities, which specialize to a result of Garsia and Gessel on the generating function of the joint distribution of five permutation statistics.

Résumé. Nous définissons une action de l'algèbre de Hecke-0 de type A sur l'anneau Stanley-Reisner de l'algèbre de Boole. En étudiant cette action, on obtient une famille de fonctions symétriques non commutatives multivariées, qui se spécialisent pour les non commutatives fonctions de Hall-Littlewood symétriques et leur $(q, t)$-analogues introduits par Bergeron et Zabrocki. Nous obtenons également des identités de fonction quasisymmetric multivariées, qui se spécialisent à la suite de Garsia et Gessel sur la fonction génératrice de la distribution conjointe de cinq statistiques de permutation.
\end{abstract}

Keywords: 0-Hecke algebra, Stanley-Reisner ring, Boolean algebra, noncommutative Hall-Littlewood symmetric function, multivariate quasisymmetric function.

\section{Introduction}

Let $\mathbb{F}$ be any field. The symmetric group $\mathfrak{S}_{n}$ naturally acts on the polynomial ring $\mathbb{F}[X]:=\mathbb{F}\left[x_{1}, \ldots, x_{n}\right]$ by permuting the variables $x_{1}, \ldots, x_{n}$. The invariant algebra $\mathbb{F}[X]^{\mathfrak{S}_{n}}$, which consists of all the polynomials fixed by this $\mathfrak{S}_{n}$-action, is a polynomial algebra generated by the elementary symmetric functions $e_{1}, \ldots, e_{n}$. The coinvariant algebra $\mathbb{F}[X] /\left(\mathbb{F}[X]_{+}^{\mathfrak{S}_{n}}\right)$, with $\left(\mathbb{F}[X]_{+}^{\mathfrak{S}_{n}}\right)=\left(e_{1}, \ldots, e_{n}\right)$, is a vector space of dimension $n$ ! over $\mathbb{F}$, and when $\mathbb{F}$ has characteristic larger than $n$ the coinvariant algebra carries the regular representation of $\mathfrak{S}_{n}$. A well known basis for $\mathbb{F}[X] /\left(\mathbb{F}[X]_{+}^{\mathfrak{S}_{n}}\right)$ consists of the descent monomials. Garsia [7] obtained this basis by transferring a natural basis from the Stanley-Reisner ring $\mathbb{F}\left[\mathcal{B}_{n}\right]$ of the Boolean algebra $\mathcal{B}_{n}$ to the polynomial ring $\mathbb{F}[X]$. Here the Boolean algebra $\mathcal{B}_{n}$ is the set of all subsets of $[n]:=\{1,2, \ldots, n\}$ partially ordered by inclusion, and the Stanley-Reisner ring $\mathbb{F}\left[\mathcal{B}_{n}\right]$ is the quotient of the polynomial algebra $\mathbb{F}\left[y_{A}: A \subseteq[n]\right]$ by the ideal $\left(y_{A} y_{B}: A\right.$ and $B$ are incomparable in $\left.\mathcal{B}_{n}\right)$.

The 0-Hecke algebra $H_{n}(0)$ (of type $A$ ) is a deformation of the group algebra of $\mathfrak{S}_{n}$. It acts on $\mathbb{F}[X]$ by the Demazure operators, also known as the isobaric divided difference operators, having the same

*Email: jiahuangustc@gmail.com. Supported by NSF grant DMS-1001933.

1365-8050 @ 2014 Discrete Mathematics and Theoretical Computer Science (DMTCS), Nancy, France 
invariant algebra as the $\mathfrak{S}_{n}$-action on $\mathbb{F}[X]$. In our earlier work [13], we showed that the coinvariant algebra $\mathbb{F}[X] /\left(\mathbb{F}[X]_{+}^{\mathfrak{S}_{n}}\right)$ is also isomorphic to the regular representation of $H_{n}(0)$, for any field $\mathbb{F}$, by constructing another basis for $\mathbb{F}[X] /\left(\mathbb{F}[X]_{+}^{\mathfrak{S}_{n}}\right)$ which consists of certain polynomials whose leading terms are the descent monomials. This and the previously mentioned connection between the Stanley-Reisner ring $\mathbb{F}\left[\mathcal{B}_{n}\right]$ and the polynomial ring $\mathbb{F}[X]$ motivate us to define an $H_{n}(0)$-action on $\mathbb{F}\left[\mathcal{B}_{n}\right]$.

It turns out that our $H_{n}(0)$-action on $\mathbb{F}\left[\mathcal{B}_{n}\right]$ has similar properties to the $H_{n}(0)$-action on $\mathbb{F}[X]$. It preserves an $\mathbb{N}^{n+1}$-multigrading of $\mathbb{F}\left[\mathcal{B}_{n}\right]$ and has invariant algebra equal to a polynomial algebra $\mathbb{F}[\Theta]$, where $\Theta$ is the set of rank polynomials $\theta_{i}$ (the usual analogue of $e_{i}$ in $\mathbb{F}\left[\mathcal{B}_{n}\right]$ ). We show that the $H_{n}(0)$ action is $\Theta$-linear and thus descends to the coinvariant algebra $\mathbb{F}\left[\mathcal{B}_{n}\right] /(\Theta)$. Using the descent monomials in $\mathbb{F}\left[\mathcal{B}_{n}\right]$ it is not hard to see that $\mathbb{F}\left[\mathcal{B}_{n}\right] /(\Theta)$ carries the regular representation of $H_{n}(0)$.

It is well known that every finite dimensional (complex) $\mathfrak{S}_{n}$-representation is a direct sum of simple (i.e. irreducible) $\mathfrak{S}_{n}$-modules, and the simple $\mathfrak{S}_{n}$-modules are indexed by partitions $\lambda$ of $n$, which correspond to the Schur functions $s_{\lambda}$ via the Frobenius characteristic map. Hotta-Springer [11] and Garsia-Procesi [9] discovered that the cohomology ring of the Springer fiber indexed by a partition $\mu$ of $n$ is isomorphic to certain quotient ring $R_{\mu}$ of $\mathbb{F}[X]$, which admits a graded $\mathfrak{S}_{n}$-module structure corresponding to the modified Hall-Littlewood symmetric function $\widetilde{H}_{\mu}(x ; t)$ via the Frobenius characteristic map. The coinvariant algebra of $\mathfrak{S}_{n}$ is nothing but $R_{1^{n}}$.

In our previous work [13] we established a partial analogue of the above result by showing that the $H_{n}(0)$-action on $\mathbb{F}[X]$ descends to $R_{\mu}$ if and only if $\mu=\left(1^{k}, n-k\right)$ is a hook, and if so then $R_{\mu}$ has graded quasisymmetric characteristic equal to $\widetilde{H}_{\mu}(x ; t)$ and graded noncommutative characteristic $\widetilde{\mathbf{H}}_{\mu}(\mathbf{x} ; t)$. Here $\widetilde{\mathbf{H}}_{\alpha}(\mathbf{x} ; t)$ is the noncommutative modified Hall-Littlewood symmetric function introduced by Bergeron and Zabrocki [3] for any composition $\alpha$ of $n$. Using an analogue of the nabla operator Bergeron and Zabrocki [3] also introduced a $(q, t)$-analogue $\widetilde{\mathbf{H}}_{\alpha}(\mathbf{x} ; q, t)$ for any composition $\alpha$. Now we provide in Theorem 1.1 below a complete representation theoretic interpretation for $\widetilde{\mathbf{H}}_{\alpha}(\mathbf{x} ; t)$ and $\widetilde{\mathbf{H}}_{\alpha}(\mathbf{x} ; q, t)$ by the $H_{n}(0)$-action on $\mathbb{F}\left[\mathcal{B}_{n}\right]$.

To state our result, we first recall the two characteristic maps for representations of $H_{n}(0)$ introduced by Krob and Thibon [14], which we call the quasisymmetric characteristic and the noncommutative characteristic. The simple $H_{n}(0)$-modules are indexed by compositions $\alpha$ of $n$ and correspond to the fundamental quasisymmetric functions $F_{\alpha}$ via the quasisymmetric characteristic; the projective indecomposable $H_{n}(0)$-modules are also indexed by compositions $\alpha$ of $n$ and correspond to the noncommutative ribbon Schur functions $\mathbf{s}_{\alpha}$ via the noncommutative characteristic. See $\$ 2$ for details.

Theorem 1.1 Let $\alpha$ be a composition of $n$. Then there exists a homogeneous $H_{n}(0)$-invariant ideal $I_{\alpha}$ of the multigraded algebra $\mathbb{F}\left[\mathcal{B}_{n}\right]$ such that the quotient algebra $\mathbb{F}\left[\mathcal{B}_{n}\right] / I_{\alpha}$ becomes a projective $H_{n}(0)$ module with multigraded noncommutative characteristic equal to

$$
\widetilde{\mathbf{H}}_{\alpha}\left(\mathbf{x} ; t_{1}, \ldots, t_{n-1}\right):=\sum_{\beta \preccurlyeq \alpha} \underline{t}^{D(\beta)} \mathbf{s}_{\beta} \quad \text { inside } \quad \operatorname{NSym}\left[t_{1}, \ldots, t_{n-1}\right] .
$$

One has $\widetilde{\mathbf{H}}_{\alpha}\left(\mathbf{x} ; t, t^{2}, \ldots, t^{n-1}\right)=\widetilde{\mathbf{H}}_{\alpha}(\mathbf{x} ; t)$, and obtains $\widetilde{\mathbf{H}}_{\alpha}(\mathbf{x} ; q, t)$ from $\widetilde{\mathbf{H}}_{1^{n}}\left(\mathbf{x} ; t_{1}, \ldots, t_{n-1}\right)$ by taking $t_{i}=t^{i}$ for all $i \in D(\alpha)$, and $t_{i}=q^{n-i}$ for all $i \in[n-1] \backslash D(\alpha)$.

Here $D(\alpha)$ is the set of partial sums of $\alpha$, the notation $\beta \preccurlyeq \alpha$ means $\alpha$ and $\beta$ are compositions of $n$ with $D(\beta) \subseteq D(\alpha)$, and $\underline{t}^{S}$ denotes the product $\prod_{i \in S} t_{i}$ over all elements $i$ in a multiset $S$, including the repeated ones. Taking $\alpha=\left(1^{n}\right)$ shows that $\mathbb{F}\left[\mathcal{B}_{n}\right] /(\Theta)$ carries the regular representation of $H_{n}(0)$. 
Specializations of $\widetilde{\mathbf{H}}_{1^{n}}\left(\mathbf{x} ; t_{1}, \ldots, t_{n-1}\right)$ include not only $\widetilde{\mathbf{H}}_{\alpha}(\mathbf{x} ; q, t)$, but also a more general family of noncommutative symmetric functions depending on parameters associated with paths in binary trees introduced recently by Lascoux, Novelli, and Thibon [15].

Next we study the quasisymmetric characteristic of $\mathbb{F}\left[\mathcal{B}_{n}\right]$. We combine the usual $\mathbb{N}^{n+1}$-multigrading of $\mathbb{F}\left[\mathcal{B}_{n}\right]$ (recorded by $\underline{t}:=t_{0}, \ldots, t_{n}$ ) with the length filtration of $H_{n}(0)$ (recorded by $q$ ) and obtain an $\mathbb{N} \times \mathbb{N}^{n+1}$-multigraded quasisymmetric characteristic for $\mathbb{F}\left[\mathcal{B}_{n}\right]$.

Theorem 1.2 The $\mathbb{N} \times \mathbb{N}^{n+1}$-multigraded quasisymmetric characteristic of $\mathbb{F}\left[\mathcal{B}_{n}\right]$ is

$$
\begin{aligned}
\mathrm{Ch}_{q, \underline{t}}\left(\mathbb{F}\left[\mathcal{B}_{n}\right]\right) & =\sum_{k \geq 0} \sum_{\alpha \in \operatorname{Com}(n, k+1)} \underline{t}^{D(\alpha)} \sum_{w \in \mathfrak{S}^{\alpha}} q^{\operatorname{inv}(w)} F_{D\left(w^{-1}\right)} \\
& =\sum_{w \in \mathfrak{S}_{n}} \frac{q^{\operatorname{inv}(w)} \underline{t}^{D(w)} F_{D\left(w^{-1}\right)}}{\prod_{0 \leq i \leq n}\left(1-t_{i}\right)} \\
& =\sum_{k \geq 0} \sum_{\mathbf{p} \in[k+1]^{n}} t_{p_{1}^{\prime}} \cdots t_{p_{k}^{\prime}} q^{\operatorname{inv}(\mathbf{p})} F_{D(\mathbf{p})} .
\end{aligned}
$$

Here we identify $F_{I}$ with $F_{\alpha}$ if $D(\alpha)=I \subseteq[n-1]$. The set $\operatorname{Com}(n, k)$ consists of all weak compositions of $n$ with length $k$, i.e. all the sequences $\alpha=\left(\alpha_{1}, \ldots, \alpha_{k}\right)$ of $k$ nonnegative integers with $|\alpha|:=\sum_{i=1}^{k} \alpha_{i}=n$. The descent multiset of the weak composition $\alpha$ is the multiset

$$
D(\alpha):=\left\{\alpha_{1}, \alpha_{1}+\alpha_{2}, \ldots, \alpha_{1}+\cdots+\alpha_{k-1}\right\} .
$$

We also define $\mathfrak{S}^{\alpha}:=\left\{w \in \mathfrak{S}_{n}: D(w) \subseteq D(\alpha)\right\}$. The set $[k+1]^{n}$ consists of all words of length $n$ on the alphabet $[k+1]$. Given $\mathbf{p}=\left(p_{1}, \ldots, p_{n}\right) \in[k+1]^{n}$, we write $p_{i}^{\prime}:=\#\left\{j: p_{j} \leq i\right\}$, $\operatorname{inv}(\mathbf{p}):=\#\left\{(i, j): 1 \leq i<j \leq n: p_{i}>p_{j}\right\}$, and $D(\mathbf{p}):=\left\{i: p_{i}>p_{i+1}\right\}$.

Let $\mathbf{p s}_{q ; \ell}\left(F_{\alpha}\right):=F_{\alpha}\left(1, q, q^{2}, \ldots, q^{\ell-1}, 0,0, \ldots\right)$. Applying the linear transformation $\sum_{\ell \geq 0} u_{1}^{\ell} \mathbf{p s}_{q_{1} ; \ell+1}$ and the specialization $t_{i}=q_{2}^{i} u_{2}$ for all $i=0,1, \ldots, n$ to Theorem 1.2 , we recover a result of Garsia and Gessel [8, Theorem 2.2] on the generating function of the joint distribution of five permutation statistics:

$$
\frac{\sum_{w \in \mathfrak{S}_{n}} q_{0}^{\operatorname{inv}(w)} q_{1}^{\operatorname{maj}\left(w^{-1}\right)} u_{1}^{\operatorname{des}\left(w^{-1}\right)} q_{2}^{\operatorname{maj}(w)} u_{2}^{\operatorname{des}(w)}}{\left(u_{1} ; q_{1}\right)_{n}\left(u_{2} ; q_{2}\right)_{n}}=\sum_{\ell, k \geq 0} u_{1}^{\ell} u_{2}^{k} \sum_{(\lambda, \mu) \in B(\ell, k)} q_{0}^{\operatorname{inv}(\mu)} q_{1}^{|\lambda|} q_{2}^{|\mu|}
$$

Here $(u ; q)_{n}:=\prod_{0 \leq i \leq n}\left(1-q^{i} u\right)$, the set $B(\ell, k)$ consists of pairs of weak compositions $\lambda=\left(\lambda_{1}, \ldots, \lambda_{n}\right)$ and $\mu=\left(\mu_{1}, \ldots, \mu_{n}\right)$ satisfying the conditions $\ell \geq \lambda_{1} \geq \cdots \geq \lambda_{n}, \max \left\{\mu_{i}: 1 \leq i \leq n\right\} \leq k$, and $\lambda_{i}=\lambda_{i+1} \Rightarrow \mu_{i} \geq \mu_{i+1}$ (such pairs $(\lambda, \mu)$ are sometimes called bipartite partitions), and $\operatorname{inv}(\mu)$ is the number of inversion pairs in $\mu$. Some further specializations of Theorem 1.2 imply identities of CarlitzMacMahon [6, 17] and Adin-Brenti-Roichman [1].

The structure of this paper is as follows. Section 2 reviews the representation theory of the 0 -Hecke algebra. Section 3 studies the Stanley-Reisner ring of the Boolean algebra. Section 4 defines a 0 -Hecke algebra action on the Stanley-Reisner ring of the Boolean algebra. The noncommutative and quasisymmetric characteristics are discussed in Section 5 and Section 6 Finally we give some remarks and questions for future research in Section 7, including a generalization to an action of the Hecke algebra of any finite Coxeter group on the Stanley-Reisner ring of the Coxeter complex. 


\section{Representation theory of the 0 -Hecke algebra}

We review the representation theory of the 0-Hecke algebra in this section. The (type A) Hecke algebra $H_{n}(q)$ is the associative $\mathbb{F}(q)$-algebra generated by $T_{1}, \ldots, T_{n-1}$ with relations

$$
\begin{cases}\left(T_{i}+1\right)\left(T_{i}-q\right)=0, & 1 \leq i \leq n-1, \\ T_{i} T_{j}=T_{j} T_{i}, & 1 \leq i, j \leq n-1,|i-j|>1, \\ T_{i} T_{i+1} T_{i}=T_{i+1} T_{i} T_{i+1}, & 1 \leq i \leq n-2 .\end{cases}
$$

It has an $\mathbb{F}(q)$-basis $\left\{T_{w}: w \in \mathfrak{S}_{n}\right\}$ where $T_{w}:=T_{i_{1}} \cdots T_{i_{k}}$ if $w=s_{i_{1}} \cdots s_{i_{k}}$ is a reduced expression.

Specializing $q=1$ gives the group algebra of $\mathfrak{S}_{n}$, with $s_{i}=\left.T_{i}\right|_{q=1}$ and $w=\left.T_{w}\right|_{q=1}$. Let $w \in \mathfrak{S}_{n}$. The length of $w$ equals $\operatorname{inv}(w):=\#\{(i, j): 1 \leq i<j \leq n, w(i)>w(j)\}$, and the descent set of $w$ is $D(w)=\{i: 1 \leq i \leq n-1, w(i)>w(i+1)\}$. We write $\operatorname{des}(w):=|D(w)|$ and maj $(w):=\sum_{i \in D(w)} i$.

Let $\alpha$ be a (weak) composition of $n$, and let $\alpha^{c}$ be the composition of $n$ with $D\left(\alpha^{c}\right)=[n-1] \backslash D(\alpha)$. The parabolic subgroup $\mathfrak{S}_{\alpha}$ is the subgroup of $\mathfrak{S}_{n}$ generated by $\left\{s_{i}: i \in D\left(\alpha^{c}\right)\right\}$. The set of all minimal $\mathfrak{S}_{\alpha}$-coset representatives is $\mathfrak{S}^{\alpha}:=\left\{w \in \mathfrak{S}_{n}: D(w) \subseteq D(\alpha)\right\}$. The descent class of $\alpha$ consists of the permutations in $\mathfrak{S}_{n}$ with descent set equal to $D(\alpha)$, and turns out to be an interval under the (left) weak order of $\mathfrak{S}_{n}$, denoted by $\left[w_{0}(\alpha), w_{1}(\alpha)\right]$. One sees that $w_{0}(\alpha)$ is the longest element of the parabolic subgroup $\mathfrak{S}_{\alpha^{c}}$, and $w_{1}(\alpha)$ is the longest element in $\mathfrak{S}^{\alpha}$ (c.f. Björner and Wachs [5, Theorem 6.2]).

Another interesting specialization of $H_{n}(q)$ is the 0 -Hecke algebra $H_{n}(0)$, with generators $\bar{\pi}_{i}=\left.T_{i}\right|_{q=0}$ for $i=1, \ldots, n-1$, and an $\mathbb{F}$-basis $\left\{\bar{\pi}_{w}=\left.T_{w}\right|_{q=0}: w \in \mathfrak{S}_{n}\right\}$. Let $\pi_{i}:=\bar{\pi}_{i}+1$. Then $\pi_{1}, \ldots, \pi_{n-1}$ form another generating set for $H_{n}(0)$, with the same relations as 2 except $\pi_{i}^{2}=\pi_{i}, 1 \leq i \leq n-1$. The element $\pi_{w}:=\pi_{i_{1}} \cdots \pi_{i_{k}}$ is well defined for any $w \in \mathfrak{S}_{n}$ with a reduced expression $w=s_{i_{1}} \cdots s_{i_{k}}$, and $\left\{\pi_{w}: w \in \mathfrak{S}_{n}\right\}$ is another $\mathbb{F}$-bases for $H_{n}(0)$. One can check that $\pi_{w}$ equals the sum of $\bar{\pi}_{u}$ over all $u$ less than or equal to $w$ in the Bruhat order of $\mathfrak{S}_{n}$. In particular, $\pi_{w_{0}(\alpha)}$ is the sum of $\bar{\pi}_{u}$ for all $u \in \mathfrak{S}_{\alpha^{c}}$.

Norton [18] decomposed the 0-Hecke algebra $H_{n}(0)$ into a direct sum of projective indecomposable submodules $\mathbf{P}_{\alpha}:=H_{n}(0) \cdot \bar{\pi}_{w_{0}(\alpha)} \pi_{w_{0}\left(\alpha^{c}\right)}$ for all $\alpha \models n$ (i.e. compositions of $n$ ). Each $\mathbf{P}_{\alpha}$ has an $\mathbb{F}$ basis $\left\{\bar{\pi}_{w} \pi_{w_{0}\left(\alpha^{c}\right)}: w \in\left[w_{0}(\alpha), w_{1}(\alpha)\right]\right\}$. Its radical $\operatorname{rad} \mathbf{P}_{\alpha}$ is the unique maximal $H_{n}(0)$-submodule spanned by $\left\{\bar{\pi}_{w} \pi_{w_{0}\left(\alpha^{c}\right)}: w \in\left(w_{0}(\alpha), w_{1}(\alpha)\right]\right\}$. Although $\mathbf{P}_{\alpha}$ itself is not necessarily simple, its top $\mathbf{C}_{\alpha}:=\mathbf{P}_{\alpha} / \operatorname{rad} \mathbf{P}_{\alpha}$ is a one-dimensional simple $H_{n}(0)$-module with the action of $H_{n}(0)$ given by

$$
\bar{\pi}_{i}= \begin{cases}-1, & \text { if } i \in D(\alpha), \\ 0, & \text { if } i \notin D(\alpha) .\end{cases}
$$

It follows from general representation theory of algebras (see e.g. [2, §I.5]) that $\left\{\mathbf{P}_{\alpha}: \alpha \models n\right\}$ and $\left\{\mathbf{C}_{\alpha}: \alpha \models n\right\}$ are the complete lists of pairwise non-isomorphic projective indecomposable and simple $H_{n}(0)$-modules, respectively.

Krob and Thibon [14] introduced a correspondence between $H_{n}(0)$-representations and the dual Hopf algebras QSym and NSym, which we review next. The Hopf algebra QSym has a free $\mathbb{Z}$-basis of fundamental quasisymmetric functions $F_{\alpha}$, and the dual Hopf algebra NSym has a dual basis of noncommutative ribbon Schur functions $\mathbf{s}_{\alpha}$, for all compositions $\alpha$.

Let $M=M_{0} \supseteq M_{1} \supseteq \cdots \supseteq M_{k} \supseteq M_{k+1}=0$ be a composition series of $H_{n}(0)$-modules with simple factors $M_{i} / M_{i+1} \cong \mathbf{C}_{\alpha^{(i)}}$ for $i=0,1, \ldots, k$. Then the quasisymmetric characteristic of $M$ is

$$
\operatorname{Ch}(M):=F_{\alpha^{(0)}}+\cdots+F_{\alpha^{(k)}} .
$$


The noncommutative characteristic of a projective $H_{n}(0)$-module $M \cong \mathbf{P}_{\alpha^{(1)}} \oplus \cdots \oplus \mathbf{P}_{\alpha^{(k)}}$ is

$$
\operatorname{ch}(M):=\mathbf{s}_{\alpha^{(1)}}+\cdots+\mathbf{s}_{\alpha^{(k)}} .
$$

It is not hard to extend these characteristic maps to $H_{n}(0)$-modules with gradings and filtrations.

\section{Stanley-Reisner ring of the Boolean algebra}

In this section we study the Stanley-Reisner ring of the Boolean algebra. The Boolean algebra $\mathcal{B}_{n}$ is the ranked poset of all subsets of $[n]:=\{1,2, \ldots, n\}$ ordered by inclusion, with minimum element $\emptyset$ and maximum element $[n]$. The rank of a subset of $[n]$ is defined as its cardinality. The Stanley-Reisner ring $\mathbb{F}\left[\mathcal{B}_{n}\right]$ of the Boolean algebra $\mathcal{B}_{n}$ is the quotient of the polynomial algebra $\mathbb{F}\left[y_{A}: A \subseteq[n]\right]$ by the ideal $\left(y_{A} y_{B}: A\right.$ and $B$ are incomparable in $\left.\mathcal{B}_{n}\right)$. It has an $\mathbb{F}$-basis $\left\{y_{M}\right\}$ indexed by the multichains $M$ in $\mathcal{B}_{n}$, and is multigraded by the rank multisets $r(M)$ of the multichains $M$.

The symmetric group $\mathfrak{S}_{n}$ acts on the Boolean algebra $\mathcal{B}_{n}$ by permuting the integers $1, \ldots, n$. This induces an $\mathfrak{S}_{n}$-action on the Stanley-Reisner ring $\mathbb{F}\left[\mathcal{B}_{n}\right]$, preserving its multigrading. The invariant algebra $\mathbb{F}\left[\mathcal{B}_{n}\right]^{\mathfrak{S}_{n}}$ consists of all elements in $\mathbb{F}\left[\mathcal{B}_{n}\right]$ invariant under this $\mathfrak{S}_{n}$-action. One can show that $\mathbb{F}\left[\mathcal{B}_{n}\right]^{\mathfrak{S}_{n}}=\mathbb{F}[\Theta]$, where $\Theta:=\left\{\theta_{0}, \ldots, \theta_{n}\right\}$. Garsia [7] showed that $\mathbb{F}\left[\mathcal{B}_{n}\right]$ is a free $\mathbb{F}[\Theta]$-module on the basis of descent monomials

$$
Y_{w}:=\prod_{i \in D(w)} y_{\{w(1), \ldots, w(i)\}}, \quad \forall w \in \mathfrak{S}_{n}
$$

There is an analogy between the Stanley-Reisner ring $\mathbb{F}\left[\mathcal{B}_{n}\right]$ and the polynomial ring $\mathbb{F}[X]$ via the transfer map $\tau: \mathbb{F}\left[\mathcal{B}_{n}\right] \rightarrow \mathbb{F}[X]$ defined by

$$
\tau\left(y_{M}\right):=\prod_{1 \leq i \leq k} \prod_{j \in A_{i}} x_{j}
$$

for all multichains $M=\left(A_{1} \subseteq \cdots \subseteq A_{k}\right)$ in $\mathcal{B}_{n}$. It is not a ring homomorphism (e.g. $y_{\{1\}} y_{\{2\}}=0$ but $\left.x_{1} x_{2} \neq 0\right)$. Nevertheless, it induces an isomorphism $\tau: \mathbb{F}\left[\mathcal{B}_{n}\right] /\left(\theta_{0}\right) \cong \mathbb{F}[X]$ of $\mathfrak{S}_{n}$-modules. Moreover, it sends the rank polynomials $\theta_{1}, \ldots, \theta_{n}$ to the elementary symmetric polynomials $e_{1}, \ldots, e_{n}$, and sends the descent monomials $Y_{w}$ in $\mathbb{F}\left[\mathcal{B}_{n}^{*}\right]$ defined by 3 to the corresponding descent monomials in $\mathbb{F}[X]$ for all $w \in \mathfrak{S}_{n}$.

Example 3.1 The Boolean algebra $\mathcal{B}_{3}$ consists of all subsets of $\{1,2,3\}$. Its Stanley-Reisner ring $\mathbb{F}\left[\mathcal{B}_{3}\right]$ is a free $\mathbb{F}[\Theta]$-module with a basis of descent monomials $Y_{1}=1, Y_{s_{1}}=y_{2}, Y_{s_{2} s_{1}}=y_{3}, Y_{s_{2}}=y_{13}$, $Y_{s_{1} s_{2}}=y_{23}, Y_{s_{1} s_{2} s_{1}}=y_{23} y_{3}$, where $\Theta$ consists of the rank polynomials $\theta_{0}=y_{\emptyset}, \theta_{1}:=y_{1}+y_{2}+y_{3}$, $\theta_{2}:=y_{12}+y_{13}+y_{23}, \theta_{3}:=y_{123}$. The transfer map $\tau$ sends $\theta_{1}, \theta_{2}, \theta_{3}$ to $e_{1}, e_{2}, e_{3}$, and sends the six descent monomials in $\mathbb{F}\left[\mathcal{B}_{3}\right]$ to the six descent monomials $1, x_{2}, x_{3}, x_{1} x_{3}, x_{2} x_{3}, x_{2} x_{3}^{2}$ in $\mathbb{F}\left[x_{1}, x_{2}, x_{3}\right]$.

The homogeneous components of $\mathbb{F}\left[\mathcal{B}_{n}\right]$ are indexed by multisets with elements in $\{0, \ldots, n\}$, or equivalently by weak compositions $\alpha$ of $n$. The $\alpha$-homogeneous component $\mathbb{F}\left[\mathcal{B}_{n}\right]_{\alpha}$ has an $\mathbb{F}$-basis $\left\{y_{M}: r(M)=D(\alpha)\right\}$. Denote by $\operatorname{Com}(n, k)$ the set of all weak compositions of $n$ with length $k$. If $M=\left(A_{1} \subseteq \cdots \subseteq A_{k}\right)$ is a multichain of length $k$ in $\mathcal{B}_{n}$ then we set $A_{0}:=\emptyset$ and $A_{k+1}:=[n]$ by convention. Define $\alpha(M):=\left(\alpha_{1}, \ldots, \alpha_{k+1}\right)$, where $\alpha_{i}=\left|A_{i}\right|-\left|A_{i-1}\right|$ for all $i \in[k+1]$. Then 
$\alpha(M) \in \operatorname{Com}(n, k+1)$ and $D(\alpha(M))=r(M)$, i.e. $\alpha(M)$ indexes the homogeneous component containing $y_{M}$. Define $\sigma(M)$ to be the minimal element in $\mathfrak{S}_{n}$ which sends the standard multichain $\left[\alpha_{1}\right] \subseteq\left[\alpha_{1}+\alpha_{2}\right] \subseteq \cdots \subseteq\left[\alpha_{1}+\cdots+\alpha_{k}\right]$ with rank multiset $D(\alpha(M))$ to $M$. Then $\sigma(M) \in \mathfrak{S}^{\alpha(M)}$.

The map $M \mapsto(\alpha(M), \sigma(M))$ is a bijection between multichains of length $k$ in $\mathcal{B}_{n}$ and the pairs $(\alpha, \sigma)$ of $\alpha \in \operatorname{Com}(n, k+1)$ and $\sigma \in \mathfrak{S}^{\alpha}$. A short way to write down this encoding of $M$ is to insert bars at the descent positions of $\sigma(M)$. For example, the length-4 multichain $\{2\} \subseteq\{2\} \subseteq\{1,2,4\} \subseteq[4]$ in $\mathcal{B}_{4}$ is encoded by 2|| $14|3|$.

There is another way to encode the multichain $M$. Let $p_{i}(M):=\min \left\{j \in[k+1]: i \in A_{j}\right\}, 1 \leq i \leq n$. So $p_{i}(M)$ is the first position where $i$ appears in $M$. One checks that

$$
\left\{\begin{array}{l}
p_{i}(M)>p_{i+1}(M) \Leftrightarrow i \in D\left(\sigma(M)^{-1}\right), \\
p_{i}(M)=p_{i+1}(M) \Leftrightarrow i \notin D\left(\sigma(M)^{-1}\right), D\left(s_{i} \sigma(M)\right) \nsubseteq D(\alpha(M)), \\
p_{i}(M)<p_{i+1}(M) \Leftrightarrow i \notin D\left(\sigma(M)^{-1}\right), D\left(s_{i} \sigma(M)\right) \subseteq D(\alpha(M)) .
\end{array}\right.
$$

The map $M \mapsto p(M):=\left(p_{1}(M), \ldots, p_{n}(M)\right)$ is an bijection between the set of multichains with length $k$ in $\mathcal{B}_{n}$ and the set $[k+1]^{n}$ of all words of length $n$ on the alphabet $[k+1]$, for any fixed integer $k \geq 0$.

Let $p(M)=\mathbf{p}=\left(p_{1}, \ldots, p_{n}\right) \in[k+1]^{n}$. Then $\operatorname{inv}(p(M))=\operatorname{inv}(\sigma(M))$. Let $\mathbf{p}^{\prime}:=\left(p_{1}^{\prime}, \ldots, p_{k}^{\prime}\right)$ where $p_{i}^{\prime}:=\left|\left\{j: p_{j}(M) \leq i\right\}\right|=\left|A_{i}\right|$. Then the rank multiset of $M$ consists of $p_{1}^{\prime}, \ldots, p_{k}^{\prime}$. Define $D(\mathbf{p}):=\left\{i \in[n-1]: p_{i}>p_{i+1}\right\}$. For example, the multichain $3|14||2| 5$ corresponds to $\mathbf{p}=$ $(2,4,1,2,5) \in[5]^{5}$, and one has $\mathbf{p}^{\prime}=(1,3,3,4), D(2,5,1,2,4)=\{2\}$.

These two encodings (with slightly different notation) were already used by Garsia and Gessel [8] in their work on generating functions of multivariate distributions of permutation statistics.

\section{0-Hecke algebra action}

We saw an analogy between $\mathbb{F}\left[\mathcal{B}_{n}\right]$ and $\mathbb{F}[X]$ in the last section. The usual $H_{n}(0)$-action on the polynomial ring $\mathbb{F}[X]$ is via the Demazure operators

$$
\bar{\pi}_{i}(f):=\frac{x_{i+1} f-x_{i+1} s_{i} f}{x_{i}-x_{i+1}}, \quad \forall f \in \mathbb{F}[X], 1 \leq i \leq n-1 .
$$

The above definition is equivalent to

$$
\bar{\pi}_{i}\left(x_{i}^{a} x_{i+1}^{b} m\right)= \begin{cases}\left(x_{i}^{a-1} x_{i+1}^{b+1}+x_{i}^{a-2} x_{i+1}^{b+2} \cdots+\underline{x_{i}^{b} x_{i+1}^{a}}\right) m, & \text { if } a>b, \\ 0, & \text { if } a=b, \\ -\left(\underline{x_{i}^{a} x_{i+1}^{b}}+x_{i}^{a+1} x_{i+1}^{b-1}+\cdots+x_{i}^{b-1} x_{i+1}^{a+1}\right) m, & \text { if } a<b .\end{cases}
$$

Here $m$ is any monomial in $\mathbb{F}[X]$ containing neither $x_{i}$ nor $x_{i+1}$. Denote by $\bar{\pi}_{i}^{\prime}$ the operator obtained from (6) by taking only the leading term (underlined) in the lexicographic order of the result. Then $\bar{\pi}_{1}^{\prime}, \ldots, \bar{\pi}_{n-1}^{\prime}$ realize another $H_{n}(0)$-action on $\mathbb{F}[X]$. We call it the transferred $H_{n}(0)$-action because it can be obtained by applying the transfer map $\tau$ to our $H_{n}(0)$-action on $\mathbb{F}\left[\mathcal{B}_{n}\right]$, which we now define.

Let $M=\left(A_{1} \subseteq \cdots \subseteq A_{k}\right)$ be a multichain in $\mathcal{B}_{n}$. Recall that $p_{i}(M):=\min \left\{j \in[k+1]: i \in A_{j}\right\}$, $1 \leq i \leq n$. We define

$$
\bar{\pi}_{i}\left(y_{M}\right):= \begin{cases}-y_{M}, & p_{i}(M)>p_{i+1}(M) \\ 0, & p_{i}(M)=p_{i+1}(M) \\ s_{i}\left(y_{M}\right), & p_{i}(M)<p_{i+1}(M)\end{cases}
$$


for $i=1, \ldots, n-1$. Applying the transfer map $\tau$ one recovers $\bar{\pi}_{i}^{\prime}$. For instance, when $n=4$ one has

$$
\begin{aligned}
& \bar{\pi}_{1}\left(y_{1|34||2|}\right)=y_{2|34||1|}, \quad \bar{\pi}_{1}^{\prime}\left(x_{1}^{4} x_{2} x_{3}^{3} x_{4}^{3}\right)=x_{1} x_{2}^{4} x_{3}^{3} x_{4}^{3}, \\
& \bar{\pi}_{2}\left(y_{1|34||2|}\right)=-y_{1|34||2|}, \quad \bar{\pi}_{2}^{\prime}\left(x_{1}^{4} x_{2} x_{3}^{3} x_{4}^{3}\right)=-x_{1}^{4} x_{2} x_{3}^{3} x_{4}^{3} \text {, } \\
& \bar{\pi}_{3}\left(y_{1|34||2|}\right)=0, \quad \bar{\pi}_{3}^{\prime}\left(x_{1}^{4} x_{2} x_{3}^{3} x_{4}^{3}\right)=0 .
\end{aligned}
$$

One can check that $\bar{\pi}_{1}, \ldots, \bar{\pi}_{n-1}$ realize an $H_{n}(0)$-action on $\mathbb{F}\left[\mathcal{B}_{n}\right]$ preserving the multigrading of $\mathbb{F}\left[\mathcal{B}_{n}\right]$. If one set $t_{i}=t^{i}$ for $i=1, \ldots, n$, then there is an isomorphism $\mathbb{F}\left[\mathcal{B}_{n}\right] /(\emptyset) \cong \mathbb{F}[X]$ of graded $H_{n}(0)$-modules (which can be given explicitly, but not via the transfer map $\tau$ ).

It is not hard to show that $\mathbb{F}\left[\mathcal{B}_{n}\right]^{H_{n}(0)}=\mathbb{F}[\Theta]$, where $\mathbb{F}\left[\mathcal{B}_{n}\right]^{H_{n}(0)}$ is the invariant algebra of the $H_{n}(0)$ action on $\mathbb{F}\left[\mathcal{B}_{n}\right]$, defined as

$$
\mathbb{F}\left[\mathcal{B}_{n}\right]^{H_{n}(0)}:=\left\{f \in \mathbb{F}\left[\mathcal{B}_{n}\right]: \pi_{i} f=f, i=1, \ldots, n-1\right\} .
$$

Proposition 4.1 The $H_{n}(0)$-action on $\mathbb{F}\left[\mathcal{B}_{n}\right]$ is $\Theta$-linear.

Therefore the coinvariant algebra $\mathbb{F}\left[\mathcal{B}_{n}\right] /(\Theta)$ is a multigraded $H_{n}(0)$-module, which is isomorphic to the regular representation of $H_{n}(0)$ by Theorem 1.1 . This cannot be obtained simply by applying the transfer map $\tau$, since $\tau$ is not a map of $H_{n}(0)$-modules.

\section{Noncommutative characteristic}

In this section we use the $H_{n}(0)$-action on the Stanley-Reisner ring $\mathbb{F}\left[\mathcal{B}_{n}\right]$ of the Boolean algebra $\mathcal{B}_{n}$ to provide a noncommutative analogue of the following remarkable result.

Theorem 5.1 (Hotta-Springer [11], Garsia-Procesi [9]) For any partition $\mu=\left(0<\mu_{1} \leq \cdots \leq \mu_{k}\right)$ of $n$, there exists an $\mathfrak{S}_{n}$-invariant ideal $J_{\mu}$ of $\mathbb{C}[X]$ such that $\mathbb{C}[X] / J_{\mu}$ is isomorphic to the cohomology ring of the Springer fiber indexed by $\mu$ and has graded Frobenius characteristic equal to the modified Hall-Littlewood symmetric function

$$
\widetilde{H}_{\mu}(X ; t)=\sum_{\lambda} t^{n(\mu)} K_{\lambda \mu}\left(t^{-1}\right) s_{\lambda} \quad \text { inside } \quad \operatorname{Sym}[t]
$$

where $n(\mu):=\mu_{k-1}+2 \mu_{k-2}+\cdots+(k-1) \mu_{1}$ and $K_{\lambda \mu}(t)$ is the Kostka-Foulkes polynomial.

Example 5.2 Tanisaki [19] gives a construction for the ideal $J_{\mu}$. If $\mu=\left(1^{k}, n-k\right)$ is a hook then $J_{1^{k}, n-k}$ is generated by $e_{1}, \ldots, e_{k}$ and all monomials $x_{i_{1}} \cdots x_{i_{k+1}}$ with $1 \leq i_{1}<\cdots<i_{k+1} \leq n$.

Now consider a composition $\alpha=\left(\alpha_{1}, \ldots, \alpha_{\ell}\right)$. The major index of $\alpha$ is $\operatorname{maj}(\alpha):=\sum_{i \in D(\alpha)} i$. Viewing a partition $\mu=\left(0<\mu_{1} \leq \mu_{2} \leq \cdots\right)$ as a composition one has $\operatorname{maj}(\mu)=n(\mu)$. Recall that $\overleftarrow{\alpha}:=\left(\alpha_{\ell}, \ldots, \alpha_{1}\right)$ and $\alpha^{c}$ is the composition of $n$ with $D\left(\alpha^{c}\right)=[n-1] \backslash D(\alpha)$. We define $\alpha^{\prime}:=\overleftarrow{\alpha^{c}}=(\overleftarrow{\alpha})^{c}$. One can identify $\alpha$ with a ribbon diagram, i.e. a connected skew Young diagram without 2 by 2 boxes, which has row lengths $\alpha_{1}, \ldots, \alpha_{\ell}$, ordered from bottom to top. Note that a ribbon diagram is a Young diagram if and only if it is a hook. One can check that $\alpha^{\prime}$ is the transpose of $\alpha$; see the example below.
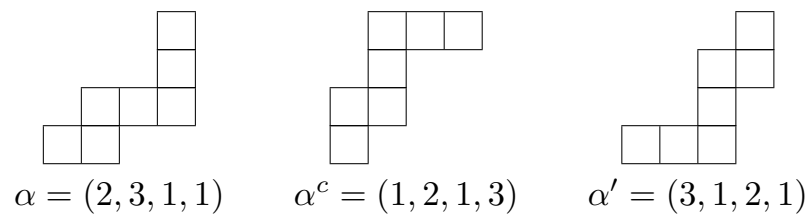
Bergeron and Zabrocki [3] introduced a noncommutative modified Hall-Littlewood symmetric function

$$
\widetilde{\mathbf{H}}_{\alpha}(\mathbf{x} ; t):=\sum_{\beta \preccurlyeq \alpha} t^{\operatorname{maj}(\beta)} \mathbf{s}_{\beta} \quad \text { inside } \quad \mathbf{N S y m}[t]
$$

and a $(q, t)$-analogue

$$
\widetilde{\mathbf{H}}_{\alpha}(\mathbf{x} ; q, t):=\sum_{\beta \models n} t^{c(\alpha, \beta)} q^{c\left(\alpha^{\prime}, \overleftarrow{\beta}\right)} \mathbf{s}_{\beta} \quad \text { inside } \quad \mathbf{N S y m}[q, t]
$$

for every composition $\alpha$, where $\mathbf{s}_{\beta}$ is the noncommutative ribbon Schur function indexed by $\beta$, and $c(\alpha, \beta):=\sum_{i \in D(\alpha) \cap D(\beta)} i$. In our earlier work [13] we provided a partial representation theoretic interpretation for $\widetilde{\mathbf{H}}_{\alpha}(\mathbf{x} ; t)$ when $\alpha=\left(1^{k}, n-k\right)$ is a hook, using the $H_{n}(0)$-action on the polynomial ring $\mathbb{F}[X]$ by the Demazure operators.

Theorem 5.3 ([13]) The ideal $J_{\mu}$ of $\mathbb{F}[X]$ is $H_{n}(0)$-invariant if and only if $\mu=\left(1^{n-k}, k\right)$ is a hook, and if that holds then $\mathbb{F}[X] / J_{\mu}$ becomes a graded projective $H_{n}(0)$-module with

$$
\begin{aligned}
& \mathbf{c h}_{t}\left(\mathbb{F}[X] / J_{\mu}\right)=\widetilde{\mathbf{H}}_{\mu}(\mathbf{x} ; t), \\
& \mathrm{Ch}_{t}\left(\mathbb{F}[X] / J_{\mu}\right)=\widetilde{H}_{\mu}(\mathbf{x} ; t) .
\end{aligned}
$$

Now we switch to the Stanley-Reisner ring $\mathbb{F}\left[\mathcal{B}_{n}\right]$ and define $I_{\alpha}$ to be its ideal generated by

$$
\Theta_{\alpha}:=\left\{\theta_{i}: i \in D(\alpha) \cup\{n\}\right\} \quad \text { and } \quad\left\{y_{A}: A \subseteq[n],|A| \notin D(\alpha) \cup\{n\}\right\}
$$

for any composition $\alpha$ of $n$. The following result is a restatement of Theorem 1.1 .

Theorem 5.4 Let $\alpha$ be a composition of $n$. Then $\mathbb{F}\left[\mathcal{B}_{n}\right] / I_{\alpha}$ is a projective $H_{n}(0)$-module with multigraded noncommutative characteristic equal to

$$
\widetilde{\mathbf{H}}_{\alpha}\left(\mathbf{x} ; t_{1}, \ldots, t_{n-1}\right):=\sum_{\beta \preccurlyeq \alpha} \underline{t}^{D(\beta)} \mathbf{s}_{\beta} \quad \text { inside } \quad \operatorname{NSym}\left[t_{1}, \ldots, t_{n-1}\right] .
$$

One has $\widetilde{\mathbf{H}}_{\alpha}\left(\mathbf{x} ; t, t^{2}, \ldots, t^{n-1}\right)=\widetilde{\mathbf{H}}_{\alpha}(\mathbf{x} ; t)$, and one obtains $\widetilde{\mathbf{H}}_{\alpha}(\mathbf{x} ; q, t)$ from $\widetilde{\mathbf{H}}_{1^{n}}\left(\mathbf{x} ; t_{1}, \ldots, t_{n-1}\right)$ by taking $t_{i}=t^{i}$ for all $i \in D(\alpha)$, and $t_{i}=q^{n-i}$ for all $i \in[n-1] \backslash D(\alpha)$.

Proof: There is an $\mathbb{F}$-basis for $\mathbb{F}\left[\mathcal{B}_{\alpha}\right] /\left(\Theta_{\alpha}\right)$ given by the descent monomials $Y_{w}$ defined in 3 for all $w \in \mathfrak{S}^{\alpha}$. The result follows from the $H_{n}(0)$-action on this basis and 4 .

The proof of this theorem is actually simpler than the proof of our partial interpretation for $\widetilde{\mathbf{H}}_{\alpha}(\mathbf{x} ; t)$ in [13]. This is because $\bar{\pi}_{i}$ sends a descent monomial in $\mathbb{F}\left[\mathcal{B}_{n}\right]$ to either 0 or \pm 1 times a descent monomial, but sends a descent monomial in $\mathbb{F}[X]$ to a polynomial in general (whose leading term is still a descent monomial). We view the Stanley-Reisner ring $\mathbb{F}\left[\mathcal{B}_{n}\right]\left(\right.$ or $\mathbb{F}\left[\mathcal{B}_{n}\right] /(\emptyset)$ ) as a $q=0$ analogue of the polynomial ring $\mathbb{F}[X]$. For an odd (i.e. $q=-1$ ) analogue, see Lauda and Russell [16].

Remark 5.5 If $\alpha=\left(1^{k}, n-k\right)$ is a hook, one can check that the ideal $I_{1^{k}, n-k}$ of $\mathbb{F}\left[\mathcal{B}_{n}\right]$ has generators $\theta_{1}, \ldots, \theta_{k}$ and all $y_{A}$ with $A \subseteq[n]$ and $|A| \notin[k]$. By Example 5.2. the images of these generators under the transfer map $\tau$ are the Tanisaki generators for the ideal $J_{1^{k}, n-k}$ of $\mathbb{F}[X]$, but $\tau\left(I_{1^{k}, n-k}\right) \neq J_{1^{k}, n-k}$. 
For any composition $\alpha \models n$, one can view $\widetilde{\mathbf{H}}_{\alpha}\left(\mathbf{x} ; t_{1}, \ldots, t_{n-1}\right)$ as a modified version of

$$
\mathbf{H}_{\alpha}=\mathbf{H}_{\alpha}\left(\mathbf{x} ; t_{1}, \ldots, t_{n-1}\right):=\sum_{\beta \preccurlyeq \alpha} \underline{t}^{D(\alpha) \backslash D(\beta)} \mathbf{s}_{\beta} .
$$

Below are some properties satisfied by $\mathbf{H}_{\alpha}$, generalizing the properties of $\mathbf{H}_{\alpha}(\mathbf{x} ; t)$ given in [3].

Proposition 5.6 Let $\alpha$ and $\beta$ be two compositions.

(i) $\mathbf{H}_{\alpha}(0, \ldots, 0)=\mathbf{s}_{\alpha}, \mathbf{H}_{\alpha}(1, \ldots, 1)=\mathbf{h}_{\alpha}$.

(ii) $\bigcup_{n \geq 0}\left\{\mathbf{H}_{\alpha}: \alpha \models n\right\}$ is a basis for $\mathbf{N S y m}\left[t_{1}, t_{2}, \ldots\right]$.

(iii) $\left\langle\mathbf{H}_{\alpha}, \mathbf{H}_{\beta}\right\rangle=(-1)^{|\alpha|+\ell(\alpha)} \delta_{\alpha, \beta^{c}}$ for any pair of compositions $\alpha$ and $\beta$.

(iv)

$$
\mathbf{H}_{\alpha} \cdot \mathbf{H}_{\beta}=\sum_{\gamma \preccurlyeq \beta}\left(\prod_{i \in D(\beta) \backslash D(\gamma)}\left(t_{i}-t_{|\alpha|+i}\right)\right)\left(\mathbf{H}_{\alpha \gamma}+\left(1-t_{|\alpha|}\right) \mathbf{H}_{\alpha \triangleright \gamma}\right) .
$$

(v) If $n=|\alpha|$ and $t \mid n:=\left(t_{1}, \ldots, t_{n-1}, 1, t_{1}, \ldots, t_{n-1}, 1, \ldots\right)$ then

$$
\mathbf{H}_{\alpha}\left(\mathbf{x} ; t_{1}, \ldots, t_{n-1}\right) \mathbf{H}_{\beta}(\mathbf{x} ; t \mid n)=\mathbf{H}_{\alpha \beta}(t \mid n) .
$$

\section{Quasisymmetric characteristic}

Now we study the quasisymmetric characteristic of $\mathbb{F}\left[\mathcal{B}_{n}\right]$. The following lemma follows easily from (4).

Lemma 6.1 Let $\alpha$ be a weak composition of $n$. Then the $\alpha$-homogeneous component $\mathbb{F}\left[\mathcal{B}_{n}\right]_{\alpha}$ of the Stanley-Reisner ring $\mathbb{F}\left[\mathcal{B}_{n}\right]$ is an $H_{n}(0)$-submodule of $\mathbb{F}\left[\mathcal{B}_{n}\right]$ with homogeneous multigrading $\underline{t}^{D(\alpha)}$ and isomorphic to the cyclic module $H_{n}(0) \pi_{w_{0}\left(\alpha^{c}\right)}$.

Since $\mathbb{F}\left[\mathcal{B}_{n}\right]_{\alpha}$ is a cyclic multigraded $H_{n}(0)$-module, we get an $\mathbb{N} \times \mathbb{N}^{n+1}$-multigraded quasisymmetric characteristic

$$
\mathrm{Ch}_{q, \underline{t}}\left(\mathbb{F}\left[\mathcal{B}_{n}\right]_{\alpha}\right)=\sum_{w \in \mathfrak{S}^{\alpha}} q^{\operatorname{inv}(w)} \underline{t}^{D(\alpha)} F_{D\left(w^{-1}\right)}
$$

where $q$ keeps track of the length filtration and $\underline{t}$ keeps track of the multigrading of $\mathbb{F}\left[\mathcal{B}_{n}\right]_{\alpha}$. This defines an $\mathbb{N} \times \mathbb{N}^{n+1}$-multigraded quasisymmetric characteristic for the Stanley-Reisner ring $\mathbb{F}\left[\mathcal{B}_{n}\right]$, which is explicitly given in Theorem 1.2 and restated below.

Theorem 6.2 The $\mathbb{N} \times \mathbb{N}^{n+1}$-multigraded quasisymmetric characteristic of $\mathbb{F}\left[\mathcal{B}_{n}\right]$ is

$$
\begin{aligned}
\mathrm{Ch}_{q, \underline{t}}\left(\mathbb{F}\left[\mathcal{B}_{n}\right]\right) & =\sum_{k \geq 0} \sum_{\alpha \in \operatorname{Com}(n, k+1)} \underline{t}^{D(\alpha)} \sum_{w \in \mathfrak{S}^{\alpha}} q^{\operatorname{inv}(w)} F_{D\left(w^{-1}\right)} \\
& =\sum_{w \in \mathfrak{S}_{n}} \frac{q^{\operatorname{inv}(w)} \underline{t}^{D(w)} F_{D\left(w^{-1}\right)}}{\prod_{0 \leq i \leq n}\left(1-t_{i}\right)} \\
& =\sum_{k \geq 0} \sum_{\mathbf{p} \in[k+1]^{n}} t_{p_{1}^{\prime}} \cdots t_{p_{k}^{\prime}} q^{\operatorname{inv}(\mathbf{p})} F_{D(\mathbf{p})} .
\end{aligned}
$$


Proof: Use the two encodings of the multichains in $\mathcal{B}_{n}$ as well as the free $\mathbb{F}[\Theta]$-basis $\left\{Y_{w}: w \in \mathfrak{S}_{n}\right\}$ of descent monomials for $\mathbb{F}\left[\mathcal{B}_{n}\right]$ discussed in Section 3

Next we explain here how this theorem specializes to (1), a result of Garsia and Gessel [8, Theorem 2.2] on the multivariate generating function of the permutation statistics $\operatorname{inv}(w), \operatorname{maj}(w), \operatorname{des}(w), \operatorname{maj}\left(w^{-1}\right)$, and $\operatorname{des}\left(w^{-1}\right)$ for all $w \in \mathfrak{S}_{n}$. First recall that

$$
F_{\alpha}=\sum_{\substack{i_{1} \geq \cdots \geq i_{n} \geq 1 \\ i \in D(\alpha) \Rightarrow i_{j}>i_{j+1}}} x_{i_{1}} \cdots x_{i_{n}}, \quad \forall \alpha \models n .
$$

Let $\mathbf{p s}_{q ; \ell}\left(F_{\alpha}\right):=F_{\alpha}\left(1, q, q^{2}, \ldots, q^{\ell-1}, 0,0, \ldots\right)$, and $(u ; q)_{n}:=(1-u)(1-q u)\left(1-q^{2} u\right) \cdots\left(1-q^{n} u\right)$. It is not hard to check (see Gessel and Reutenauer [10, Lemma 5.2]) that

$$
\sum_{\ell \geq 0} u^{\ell} \mathbf{p s}_{q ; \ell+1}\left(F_{\alpha}\right)=\frac{q^{\operatorname{maj}(\alpha)} u^{\operatorname{des}(\alpha)}}{(u ; q)_{n}} .
$$

Then applying the linear transformation $\sum_{\ell \geq 0} u_{1}^{\ell} \mathbf{p s}_{q_{1} ; \ell+1}$ and the specialization $t_{i}=q_{2}^{i} u_{2}$ for all $i=$ $0,1, \ldots, n$ to Theorem 6.2 we recover (1).

A further specialization of Theorem 6.2 gives a well known result which is often attributed to Carlitz [6] but actually dates back to MacMahon [17, Volume 2, Chapter 4].

Corollary 6.3 (Carlitz-MacMahon) Let $[k+1]_{q}:=1+q+q^{2}+\cdots+q^{k}$. Then

$$
\frac{\sum_{w \in \mathfrak{S}_{n}} q^{\operatorname{maj}(w)} u^{\operatorname{des}(w)}}{(u ; q)_{n}}=\sum_{k \geq 0}\left([k+1]_{q}\right)^{n} u^{k} .
$$

Theorem 6.2 also implies the following result, which was obtained by Adin, Brenti, and Roichman [1] from the Hilbert series of the coinvariant algebra $\mathbb{F}[X] /\left(\mathbb{F}[X]_{+}^{\mathfrak{S}_{n}}\right)$.

Corollary 6.4 (Adin-Brenti-Roichman) Let $\operatorname{Par}(n)$ be the set of weak partitions $\lambda=\left(\lambda_{1}, \ldots, \lambda_{n}\right)$ with $\lambda_{1} \geq \cdots \geq \lambda_{n} \geq 0$, and let $m(\lambda)=\left(m_{0}(\lambda), m_{1}(\lambda), \ldots\right)$, where $m_{j}(\lambda):=\#\left\{1 \leq i \leq n: \lambda_{i}=j\right\}$. Then

$$
\sum_{\lambda \in \operatorname{Par}(n)}\left(\begin{array}{c}
n \\
m(\lambda)
\end{array}\right) \prod_{i=1}^{n} q_{i}^{\lambda_{i}}=\frac{\sum_{w \in \mathfrak{S}_{n}} \prod_{i \in D(w)} q_{1} \cdots q_{i}}{\left(1-q_{1}\right)\left(1-q_{1} q_{2}\right) \cdots\left(1-q_{1} \cdots q_{n}\right)} .
$$

\section{Remarks and questions for future research}

\subsection{Hecke algebra action}

It is well known that the symmetric group $\mathfrak{S}_{n}$ is the Coxeter group of type $A_{n-1}$. The Stanley-Reisner ring of $\mathcal{B}_{n}$ is essentially the Stanley-Reisner ring of the Coxeter complex of $\mathfrak{S}_{n}$. The Hecke algebra $H_{W}(q)$ can be defined for any finite Coxeter group $W$. We can generalize our action $H_{n}(0)$-action on $\mathbb{F}\left[\mathcal{B}_{n}\right]$ to an $H_{W}(q)$-action on the Stanley-Reisner ring $\mathbb{F}(q)[\Delta(W)]$ of the Coxeter complex $\Delta(W)$ of any finite Coxeter group $W$. We show similar results for this $H_{W}(q)$-action. 


\subsection{Gluing the group algebra and the 0-Hecke algebra}

The group algebra $\mathbb{F} W$ of a finite Coxeter group $W$ naturally admits both actions of $W$ and $H_{W}(0)$. Hivert and Thiéry [12] defined the Hecke group algebra of $W$ by gluing these two actions. In type $A$, one can also glue the usual actions of $\mathfrak{S}_{n}$ and $H_{n}(0)$ on the polynomial ring $\mathbb{F}[X]$, but the resulting algebra is different from the Hecke group algebra of $\mathfrak{S}_{n}$.

Now one has a $W$-action and an $H_{W}(0)$-action on the Stanley-Reisner ring $\mathbb{F}[\Delta(W)]$. What can we say about the algebra generated by the operators $s_{i}$ and $\bar{\pi}_{i}$ on $\mathbb{F}[\Delta(W)]$ ? Is it the same as the Hecke group algebra of $W$ ? If not, what properties (dimension, bases, presentation, simple and projective indecomposable modules, etc.) does it have?

\subsection{Tits Building}

Let $\Delta(G)$ be the Tits building of the general linear group $G=G L\left(n, \mathbb{F}_{q}\right)$ and its usual BN-pair over a finite field $\mathbb{F}_{q}$; see e.g. Björner [4]. The Stanley-Reisner ring $\mathbb{F}[\Delta(G)]$ is a $q$-analogue of $\mathbb{F}\left[\mathcal{B}_{n}\right]$. The nonzero monomials in $\mathbb{F}[\Delta(G)]$ are indexed by multiflags of subspaces of $\mathbb{F}_{q}^{n}$, and there $\operatorname{are} q^{\operatorname{inv}(w)}$ many multiflags corresponding to a given multichain $M$ in $\mathcal{B}_{n}$, where $w=\sigma(M)$. Can one obtain the multivariate quasisymmetric function identities in Theorem 1.2 by defining a nice $H_{n}(0)$-action on $\mathbb{F}[\Delta(G)]$ ?

\section{Acknowledgements}

The author is grateful to Victor Reiner for providing valuable suggestions. He also thanks Ben Braun and Jean-Yves Thibon for helpful conversations and email correspondence.

\section{References}

[1] R. Adin, F. Brenti, and Y. Roichman, Descent representations and multivariate statistics, Trans. Amer. Math. Soc. 357 (2005) 3051-3082.

[2] I. Assem, D. Simson, and A. Skowroński, Elements of the representation theory of associative algebras, vol. 1: Techniques of representation theory, London Mathematical Society Student Texts, vol. 65, Cambridge University Press, Cambridge, 2006.

[3] N. Bergeron and M. Zabrocki, $q$ and $q, t$-analogs of non-commutative symmetric functions, Discrete Math. 298 (2005) 79-103.

[4] A. Björner, Some combinatorial and algebraic properties of Coxeter complexes and Tits buildings, Adv. Math. 52 (1984) 173-212.

[5] A. Björner and M. Wachs, Generalized quotients in Coxeter groups, Trans. Amer. Math. Soc. 308 (1988) 1-37.

[6] L. Carlitz, A combinatorial property of $q$-Eulerian numbers, Amer. Monthly, 82 (1975) 51-54.

[7] A. Garsia, Combinatorial methods in the theory of Cohen-Macaulay rings, Adv. Math. 38 (1980) 229-266. 
[8] A. Garsia and I. Gessel, Permutation statistics and partitions, Adv. Math. 31 (1979) 288-305.

[9] A. Garsia and C. Procesi, On certain graded $S_{n}$-modules and the $q$-Kostka polynomials, Adv. Math. 94 (1992) 82-138.

[10] I. Gessel and C. Reutenauer, Counting permutations with given cycle structure and descent set, J. Combin. Theory Ser. A 64 (1993) 189-215.

[11] R. Hotta and T. A. Springer, A specialization theorem for certain Weyl group representations and an application to the Green polynomials of unitary groups, Invent. Math. 41 (1977), 113-127.

[12] F. Hivert and N. Thiéry, The Hecke group algebra of a Coxeter group and its representation theory, J. Algebra, 321(2009) 2230-2258.

[13] J. Huang, 0-Hecke algebra actions on coinvariants and flags, to appear in J. Algebraic Combin..

[14] D. Krob and J.-Y. Thibon, Noncommutative symmetric functions IV: Quantum linear groups and Hecke algebras at $q=0$, J. Algebraic Combin. 6 (1997) 339-376.

[15] A. Lascoux, J.-C. Novelli, and J.-Y. Thibon, Noncommutative symmetric functions with matrix parameters, J. Algebr. Comb. 37 (2013) 621-642.

[16] A. Lauda and H. Russell, Oddification of the cohomology of type A Springer varieties, arXiv:1203.0797.

[17] P.A. MacMahon, Combinatorial Analysis, Vols. 1 and 2, Cambridge University Press 1915-1916; reprinted by Chelsea 1960.

[18] P.N. Norton, 0-Hecke algebras, J. Austral. Math. Soc. A 27 (1979) 337-357.

[19] T. Tanisaki, Defining ideals of the closures of conjugacy classes and representations of the Weyl groups, Tohoku Math. J. 34 (1982), 575-585. 